\title{
Personalidad y Autoestima: Un análisis sobre el importante papel de sus relaciones
}

\author{
Personality and Self-Esteem: An analysis of the important role of their association
}

\author{
Hugo Simkin \\ Universidad de Buenos Aires, Facultad de Psicología, Argentina \\ Marián Pérez-Marín \\ Universidad de Valencia, Facultad de Psicología, España.
}

Recibido (13/06/2014) Aceptado (27/02/2017)

\begin{abstract}
Resumen
El modelo de los cinco factores es uno de los principales enfoques para el estudio de la personalidad. Tras su surgimiento, entre los aspectos más relevantes a analizar ha sido su relación con la autoestima, en tanto ésta constituye un aspecto central del modelo. Esta relación cuenta con numerosos aportes empíricos pero sigue señalándose la necesidad de realizar estudios que ayuden a comprender su naturaleza teórica. El objetivo de este trabajo es contribuir a estas investigaciones, analizando las relaciones entre factores de personalidad y la autoestima. La muestra estuvo constituida por 576 estudiantes universitarios de entre 18-35 años de edad. De acuerdo con los resultados, la autoestima se asocia negativamente a neuroticismo y positivamente, a la extraversión, responsabilidad, amabilidad, apertura y a los factores de segundo orden estabilidad y plasticidad. Concluimos la importancia de seguir investigando para comprender mejor el lugar de la autoestima dentro del modelo y la teoría de los cinco factores.
\end{abstract}

Palabras clave: Personalidad, Autoestima, FFM, Modelo de los Cinco Factores.

\begin{abstract}
The five factor model has been established as one of the main approaches in the study of personality. After its emergence, one of the most important aspects to be analyzed has been its relationship with self-esteem, considering the central role that the latest has in the model. In spite of the large empirical support existing about this relationship, the need of a deeper understanding of its theoretical nature has been pointed out. The aim of our work joins the previous research, in analyzing the existence of relationships between personality factors and self-esteem. The sample was 576 university students, between $18-35$ years old. The present findings show that self-esteem is negatively associated with neuroticism, and positively associated with extraversion, conscientiousness, agreeableness, openness, and with second-order factors elasticity and plasticity. We conclude that further research that allow a better understanding of the role of self-esteem within the five factor model and theory should be strongly encouraged.

Keywords: Personality, Self-esteem, FFM, Five Factor Model.
\end{abstract}

Correspondencia: Hugo Simkin, hugosimkin@psi.uba.ar, Hipólito Yrigoyen 3242, Ciudad Autónoma de Buenos Aires, Argentina 


\section{Introducción}

A pesar de que la personalidad y la autoestima representan dos de los constructos más estudiados en psicología (Mruk, 2017; Rhodewalt \& Tragakis, 2003), la naturaleza teórica de su relación persiste como un tema de debate actual (Wagner \& Gerstorf, 2017; Zeigler-Hill, Besser, Myers, Southard, \& Malkin, 2013). Numerosos estudios han explorado este vínculo en distintos contextos como Alemania (Luan et al., 2018; Michaelides et al., 2016), Argentina (Helueni \& Enrique, 2015), Brasil (Paiva, Pimente, \& Moura, 2017; Schaffhuser, Wagner, Lüdtke, \& Allemand, 2014), Bélgica (Rassart, Luyckx, Moons, \& Weets, 2014), China (Luk \& Bond, 1993; Shi, Liu, Yang, \& Wang, 2015; Zeigler-Hill et al., 2015), Eslovaquia (Zitny \& Halama, 2011), Estados Unidos (Hair \& Graziano, 2003; Shackelford \& Michalski, 2011), Estonia (Kaare, Mõttus, \& Konstabel, 2009), Filipinas (Chen, Widjaja, \& Yen, 2015), Grecia (Tsigilis \& Srebauite, 2015), Inglaterra (Hills, Francis, \& Jennings, 2006), Indonesia (Chen et al., 2015), Israel (Zeigler-Hill et al., 2015), Japón (Shikishima et al., 2018), Malasia (Keng Cheng \& Law, 2015), Noruega (Halvorsen \& Heyerdahl, 2006; von Soest, Wagner, Hansen, \& Gerstorf, 2017), Portugal (Neto \& Mullet, 2004), Suecia (Lawenius \& Veisson, 1996), Taiwan (Chen et al., 2015), Tailandia (Chen et al., 2015), o Turquía (Koruklu, 2015). Por lo general, se observa que la autoestima se encuentra fuertemente asociada al neuroticismo (Bleidorn et al., 2016; Judge, Erez, Bono, \& Thoresen, 2002), moderadamente asociada a la extraversión y a la responsabilidad (Costa, McCrae, \& Dye, 1991; Digman, 1990) y débilmente asociada a la amabilidad y la apertura a la experiencia (Robins, Tracy, Trzesniewski, Potter, \& Gosling, 2001). De acuerdo con la literatura, los rasgos de la personalidad afectarían la autoestima al incrementar la autoeficacia -vale decir, la capacidad para alcanzar con éxito los objetivos propuestosy valoración por parte de la familia, el grupo de pares, las personas significativas o la comunidad en general (Mruk, 2013; Simkin \& Azzollini, 2015).

\section{Autoestima y apertura a la experiencia}

La débil relación entre la autoestima y la apertura se debería a que, dependiendo del tipo de tarea, la apertura podría promover tanto una mayor eficacia como, en algunos casos, interferir con los objetivos propuestos. Por ejemplo, se ha observado que las personas con alta apertura son capaces de elaborar ideas novedosas pero, con frecuencia, persiguen objetivos difusos o poco claros, presumiblemente porque siguen generando nuevas ideas sin concretar ninguna de ellas (Piedmont, Sherman, \& Sherman, 2012). Asimismo, una elevada apertura no representa un rasgo socialmente deseable de manera unívoca, por lo que tampoco se encuentra necesariamente asociada a una mayor valoración social. Por ejemplo, se ha sugerido que las personas prefieren características en apertura similares a las propias en las relaciones íntimas y cercanas, de modo que quienes presentan alta apertura se "aburren" con personas de baja apertura y estas últimas ven a las primeras como "pretenciosas" (Bleidorn et al., 2016; Figueredo, Sefcek, \& Jones, 2006).

\section{Autoestima y responsabilidad}

De acuerdo con McCrae y Löckenhoff (2010), las personas responsables suelen encontrar mayor discrepancia entre los resultados obtenidos y los esperados, lo que las motiva a superarse constantemente. Además, tienden a no procrastinar, perseveran y son capaces de demorar la gratificación, lo que incrementa su autoeficacia (Keng Cheng \& Law, 2015). Además, de acuerdo con Costa et al., (1991), las personas más responsables consiguen valoración social a partir del cumplimiento de sus obligaciones para con otros, comprometiéndose en sus relaciones interpersonales y presentándose leales como compañeros de trabajo.

\section{Autoestima y extraversión}

De acuerdo con la literatura, la asociación positiva entre la autoestima y la extraversión podría explicarse en parte debido a que el carácter gregario de las personas extravertidas favorecería su autoeficacia, particularmente en tareas grupales, donde se presentan como líderes atractivos y carismáticos (De Jong, Bouhuys, \& Barnhoorn, 1999). A su vez, el estilo interpersonal sociotrópico y el elevado optimismo los conducen a fortalecer sus relaciones interpersonales (Tsigilis \& Srebauite, 2015), obteniendo una mayor valoración social (Swickert, Hittner, Kitos, \& Cox-Fuenzalida, 2004).

\section{Autoestima y amabilidad}

El vínculo débil entre estos constructos podría explicarse, en primer lugar, debido a que, si bien las personas con elevada amabilidad se desenvuelven adecuadamente en el trabajo en equipo, tienden a fallar en culturas organizacionales altamente competitivas. En este sentido, es poco frecuente que 
procuren ser reconocidos por sus méritos y suelen someterse a condiciones de explotación laboral (Judge \& Cable, 1997). Además, tienen dificultades para generar acuerdos con otros, lo que afectaría el cumplimiento de objetivos (Rassart et al., 2014). En segundo lugar, la amabilidad no se encuentra necesariamente asociada a una mayor valoración social. De acuerdo con algunos autores, este conjunto de rasgos facilitaría las relaciones interpersonales fortaleciendo, en consecuencia, su percepción de valoración social (JensenCampbell, Graziano, \& West, 1995; Zeigler-Hill et al., 2015). Costa et al., (1991) observan, sin embargo, que la amabilidad por sí sola no es una característica socialmente valorada de manera positiva. A pesar de que las personas suelen preferir a individuos más amables, en ausencia de otros atributos, la amabilidad puede ser percibida como un indicador de sometimiento, rasgo socialmente valorado de manera negativa.

\section{Autoestima y neuroticismo}

Las personas con mayor neuroticismo tienden a carecer de confianza en su capacidad para llevar a cabo eficazmente las tareas requeridas, encontrando dificultades para la resolución de problemas, lo que afecta directamente su autoeficacia (Gist \& Mitchell, 1992; Koruklu, 2015). Asimismo, tienden a estar pendientes de la aceptación social (McDonald \& Leary, 2005; Schaffhuser et al., 2014), a la vez que su estilo pesimista es socialmente valorado de manera negativa (McCrae \& Costa, 2012; Schwab \& Petersen, 1990; R. J. Swickert, Hittner, \& Foster, 2010).

\section{Autoestima, estabilidad y plasticidad}

A pesar de que se han identificado asociaciones positivas entre la autoestima y los factores de segundo orden de la personalidad, la naturaleza teórica de la relación aún no ha sido abordada de manera consistente (Erdle, 2013; Erdle, Gosling, \& Potter, 2009; Şimşek, 2012). Siguiendo la literatura, el factor plasticidad -o beta-, vinculado al desarrollo personal y a la posibilidad de alcanzar objetivos y metas (Digman, 1997), podría promover una mayor autoeficacia. Por su parte, debido a que el factor estabilidad -o alfa- remite a rasgos socialmente deseables, es posible que pueda promover una mayor valoración social (DeYoung, 2006; DeYoung, Peterson, \& Higgins, 2002).

\section{Método}

\section{Participantes.}

La muestra fue no probabilística, incidental, compuesta por 576 estudiantes universitarios con edades comprendidas entre los 18 y 30 años $(M=16.57 ; D E=.54)$ y de ambos sexos (Hombres $=43.1 \%$; Mujeres $=56.9 \%)$. Los datos fueron recolectados a través de un instrumento de evaluación de índole autoadministrable.

\section{Instrumentos.}

1 - Inventario de los Cinco Grandes o Big Five Intentory (BFI) (Benet-Martínez \& John, 1998; John \& Srivastava, 1999)1991. El BFI es un cuestionario de 44 ítems que evalúan cinco dimensiones de la personalidad en población adolescente y adulta: Apertura a la experiencia, Responsabilidad, Extraversión, Amabilidad y Neuroticismo o Estabilidad Emocional. Para el presente estudio se realizó una adaptación al contexto local de la técnica, siguiendo las recomendaciones de la literatura (Castro Solano \& Casullo, 2001; Simkin, 2013). 2 - Escala de Autoestima de Rosenberg o Rosenberg SelfEsteem Scale (RSES) (Rosenberg, 1965). La RSES es un cuestionario de 10 ítems que evalúan la autoestima en población adolescente y adulta. Para el presente estudio se ha adaptado la técnica siguiendo las recomendaciones de la literatura (Baños \& Guillén, 2000; Góngora \& Casullo, 2009).

\section{Procedimiento.}

Los sujetos fueron invitados a participar de forma voluntaria, solicitándoles su consentimiento de forma escrita. A la vez, se les hizo constar que los datos se utilizarían con fines exclusivamente científicos.

\section{Análisis de datos.}

Para realizar el análisis estadístico se utilizaron los programas SPSS 22 y AMOS 21. En primer lugar, se analizó la confiabilidad de las técnicas a partir del análisis de consistencia interna. En segundo lugar, se evaluó la validez de constructo empleando un análisis factorial confirmatorio (AFC). Los modelos fueron evaluados a partir de los índices de bondad de ajuste $\chi 2$, AGFI, CFI, IFI y RMSEA. Finalmente, se analizaron las correlaciones entre ambos constructos. 


\section{Resultados}

En primer lugar, se evaluó la consistencia interna del BFI a partir del estadístico alfa de Cronbach para los factores Apertura $(\alpha=.77)$, Responsabilidad $(\alpha=.74)$, Extraversión $(\alpha=.76)$, Amabilidad $(\alpha=.68)$, y Neuroticismo $(\alpha=.78)$, el cual arroja resultados aceptables de acuerdo con la literatura (Martínez Arias, Hernández, \& Lloreda, 2006).

A continuación, se procedió a realizar el AFC del BFI. Los resultados dan cuenta buen ajuste de los datos al modelo de dos factores de orden superior (Bentler, 1990).

Tabla I: AFC BFI. Índices de ajuste

\begin{tabular}{lccccc}
\hline & X2(gl)* & AGFI & CFI & IFI & RMSEA \\
\hline $\begin{array}{l}\text { Factores de } \\
\text { orden superior }\end{array}$ & $3.69(4)$ & .99 & 1.00 & .966 & .000 \\
\hline
\end{tabular}

*. $p=.449$

En la figura I puede observarse el modelo de dos factores de orden superior de la personalidad propuesto en la literatura (DeYoung, 2006; Digman, 1997; Markon, Krueger, \& Watson, 2005).

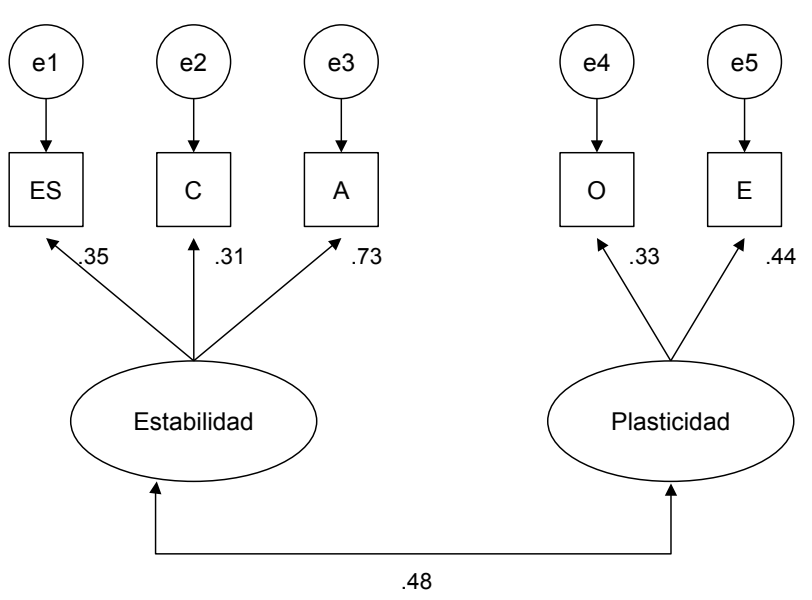

Figura I: Modelo estructural del Inventario de los Cinco Grandes

Luego, se evaluó la consistencia interna de la RSES, obteniendo guarismos aceptables, de acuerdo con la literatura $(\alpha=.85)$ (Martínez Arias et al., 2006).
Posteriormente, se llevó a cabo el AFC de la RSES, el cual da cuenta de buen ajuste de los datos al modelo unidimensional (Bentler, 1990).

Tabla II. AFC RSES. Índices de ajuste

\begin{tabular}{lccccc}
\hline & $\mathrm{X} 2(\mathrm{gl})^{*}$ & AGFI & CFI & IFI & RMSEA \\
\hline \multirow{2}{*}{ RSES } & $\begin{array}{c}216.98 \\
(35)\end{array}$ & .92 & .90 & .90 & .059 \\
\hline
\end{tabular}

*. $\mathrm{p}<.01$

En la figura II puede observarse el modelo unidimensional de la RSES.

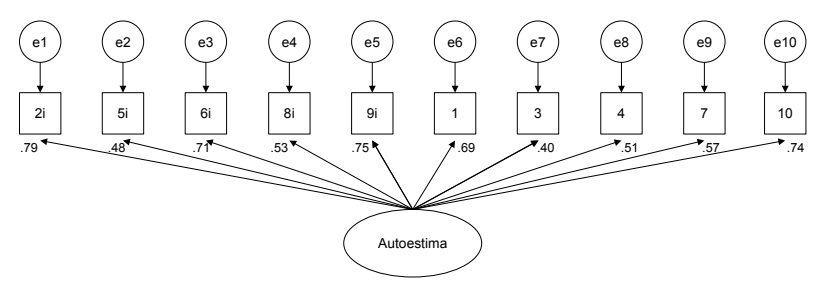

Figura II, Modelo estructural del Inventario de Autoestima de Rosenberg

Finalmente, se presentan las correlaciones entre los factores de la personalidad y la autoestima.

Tabla III. Correlaciones entre los factores de la personalidady la autoestima

\begin{tabular}{lcccccccc}
\hline 1. Estabilidad & & & & & & & \\
Emocional & - & & & & & \\
\hline 2. Apertura & .05 & - & & & & & \\
3. Responsabilidad & $.10^{*}$ & .05 & - & & & & \\
4. Extraversión & $.13^{* *}$ & $.15^{* *}$ & .02 & - & & & \\
5. Amabilidad & $.26 *$ & $.11^{* *}$ & .25 & $.15^{* *}$ & - & & \\
6, Estabilidad & $.70^{* *}$ & .08 & $.65^{* *}$ & $.15^{* *}$ & $.70^{* *}$ & - & \\
7. Plasticidad & $.12^{* *}$ & $.77^{* *}$ & .04 & $.74^{* *}$ & $.18^{* *}$ & $.15^{* *}$ & - \\
8. Autoestima & $.37^{* *}$ & $.14^{* *}$ & $.33^{* *}$ & $.22^{* *}$ & $.27^{* *}$ & $.45^{* *}$ & $.25^{* *}$ & - \\
\hline
\end{tabular}

$* * . p<.01 ; * . p<.05$

De acuerdo con los resultados, la autoestima se asocia negativamente al neuroticismo y positivamente a la apertura, responsabilidad, extraversión, amabilidad, estabilidad y plasticidad. 


\section{Discusión.}

El presente trabajo ha contribuido a explorar el modo en que los rasgos de la personalidad se asocian a la autoestima. Los resultados son consistentes con estudios anteriores, de modo que la autoestima se asocia positivamente a la apertura, la responsabilidad, la extraversión, la amabilidad, la estabilidad y la plasticidad y negativamente al neuroticismo (Erdle et al., 2009; Robins et al., 2001; Wagner \& Gerstorf, 2017). Tal como ha sido sugerido, los rasgos de la personalidad podrían afectar a la autoestima al promover la autoeficacia y la aceptación social (Mruk, 2013; Simkin \& Azzollini, 2015). La inclusión de los factores de segundo orden de la personalidad abre una serie de interrogantes acerca de la estructura factorial del constructo. Si bien distintos autores han observado que los rasgos se encuentran organizados jerárquicamente en cinco niveles (Costa \& McCrae, 1980; Piedmont \& Chae, 1997), McCrae y Costa (2012) sugieren que resultaría posible expandir el modelo a la luz de nuevas investigaciones. En este sentido, futuros estudios que procuren explorar la relación entre la personalidad y la autoestima deberían considerar incorporar al modelo los factores de segundo orden (DeYoung, 2006; Digman, 1997; Markon et al., 2005) y nuevas dimensiones (Ashton \& Lee, 2001; Cheung, Cheung, Leung, Ward, \& Leong, 2003; Piedmont, 1999). En el contexto local, se ha observado que la espiritualidad podría considerarse como un sexto factor del modelo (Simkin, 2017). A la vez, aún se requieren desarrollos teóricos aborden con mayor profundidad tanto la dimensionalidad del modelo de los cinco factores como su relación con la autoestima, especialmente en el marco de la teoría de los cinco factores (Simkin \& Azzollini, 2015; Wagner \& Gerstorf, 2017).

\section{Referencias.}

Ashton, M. C., \& Lee, K. (2001). A theoretical basis for the major dimensions of personality. European Journal of Personality, 15(5), 327-353. https://doi.org/10.1002/per.417

Baños, R. M., \& Guillén, V. (2000). Psychometric characteristics in normal and social phobic samples for a Spanish version of the Rosenberg Self-Esteem Scale. Psychological Reports, 87(1), 269-274. https://doi. org/10.2466/pr0.2000.87.1.269

Benet-Martínez, V., \& John, O. P. (1998). Los Cinco Grandes across cultures and ethnic groups: multitrait multimethod analyses of the Big Five in Spanish and English. Journal of Personality and Social Psychology, 75(3), 729-50. Retrieved from http://www.ncbi.nlm.nih. gov/pubmed/9781409
Bentler, P. M. (1990). Comparative fit indexes in structural models. Psychological Bulletin, 107(2), 238-246. https://doi.org/10.1037/00332909.107.2.238

Bleidorn, W., Schönbrodt, F., Gebauer, J. E., Rentfrow, P. J., Potter, J., \& Gosling, S. D. (2016). To Live Among Like-Minded Others. Psychological Science, 27(3), 419-427. https://doi.org/10.1177/0956797615627133

Castro Solano, A., \& Casullo, M. M. (2001). Rasgos de personalidad, bienestar psicológico y rendimiento académico en adolescentes argentinos. Interdisciplinaria, 18(1), 65-85. Retrieved from http://scholar.google. $\mathrm{com} / \mathrm{scholar}$ hl=en\&btnG=Search\&q=intitle:Rasgos + de + personali$\mathrm{dad},+$ bienestar + psicologico $+\mathrm{y}+$ rendimiento + academico + en + adolescentes+argentinos $\# 0$

Chen, J. V., Widjaja, A. E., \& Yen, D. C. (2015). Need for Affiliation, Need for Popularity, Self-Esteem, and the Moderating Effect of Big Five Personality Traits Affecting Individuals' Self-Disclosure on Facebook. International Journal of Human-Computer Interaction, 31(11), 815-831. https://doi.org/10.1080/10447318.2015.1067479

Cheung, F. M., Cheung, S. F., Leung, K., Ward, C., \& Leong, F. (2003). The English version of the Chinese Personality Assessment Inventory. Journal of Cross-Cultural Psychology, 34(4), 433-452.

Costa, P. T., \& McCrae, R. R. (1980). Still stable after all these years: Personality as a key to some issues in adulthood and old age. In P. B. Baltes \& O. G. Brim (Eds.), Life span development and behavior (Vol. 3, pp. 65-102). New York: Academic Press.

Costa, P. T., McCrae, R. R., \& Dye, D. A. (1991). Facet Scales for Agreeableness and Conscientiousness: A Revision of the NEO Personality Inventory. Personality and Individual Differences, 12(9), 887-898. https://doi.org/10.1016/0191-8869(91)90177-D

De Jong, R. D., Bouhuys, S. A., \& Barnhoorn, J. C. (1999). Personality, Self-Efficacy and Functioning in Management Teams: A Contribution to Validation. International Journal of Selection and Assessment, 7(1), 46-49.

DeYoung, C. G. (2006). Higher-order factors of the Big Five in a multi-informant sample. Journal of Personality and Social Psychology, 91(6), 1138-1151. https://doi.org/10.1037/0022-3514.91.6.1138

DeYoung, C. G., Peterson, J. B., \& Higgins, D. M. (2002). Higher-order factors of the Big Five predict conformity: Are there neuroses of health? Personality and Individual Differences, 33(4), 533-552. https://doi. org/10.1016/S0191-8869(01)00171-4

Digman, J. M. (1990). Personality Structure: Emergence of the Five-Factor Model. Annual Review of Psychology, 41, 417-440. https://doi. org/10.1146/annurev.ps.41.020190.002221

Digman, J. M. (1997). Higher-order factors of the Big Five. Journal of Personality and Social Psychology, 73(6), 1246-1256. https://doi. org/10.1037/0022-3514.73.6.1246

Erdle, S. (2013). Self-esteem and higher-order factors of personality: A reply to Simsek. Personality and Individual Differences, 54(4), 545-546. https://doi.org/10.1016/j.paid.2012.10.030 
Erdle, S., Gosling, S. D., \& Potter, J. (2009). Does self-esteem account for the higher-order factors of the Big Five? Journal of Research in Personality, 43(5), 921-922. https://doi.org/10.1016/j.jrp.2009.04.012

Figueredo, A. J., Sefcek, J. A., \& Jones, D. N. (2006). The ideal romantic partner personality. Personality and Individual Differences, 41(3), 431-441. https://doi.org/10.1016/j.paid.2006.02.004

Gist, M. E., \& Mitchell, T. B. (1992). Self-Efficacy: a Theoretical Analysis of Its Determinants and Malleability. Academy of Management Review, 17(2), 183-211. https://doi.org/10.5465/AMR.1992.4279530

Góngora, V. C., \& Casullo, M. M. (2009). Validación de la escala de autoestima de Rosenberg en población general y en población clínica de la Ciudad de Buenos Aires. Revista Iberoamericana de Diagnóstico $Y$ Evaluacion Psicológica, 27(1), 179-194. Retrieved from http://www. aidep.org/03_ridep/R27/R279.pdf

Hair, E. C., \& Graziano, W. G. (2003). Self-Esteem, Personality and Achievement in High School: A Prospective Longitudinal Study in Texas. Journal of Personality, 71(6), 971-994. https://doi.org/10.1111/14676494.7106004

Halvorsen, I., \& Heyerdahl, S. (2006). Girls with Anorexia Nervosa as Young Adults:Personality, Self-Esteem, and Life Satisfaction. International Journal of Eating Disorders, 39(4), 285-293. https://doi. org/10.1002/eat.20248

Helueni, B. R., \& Enrique, M. (2015). La Evaluación Multidimensional de la Autoestima y su relación con el Modelo de los Cinco Factores. Psocial, 1(3), 12-17.

Hills, P. R., Francis, L. J., \& Jennings, P. (2006). Religious behavior, personality, and dimensions of self-esteem among 13-to 15-year-old adolescents. Journal of Research on Christian, 15(1), 61-76. https:// doi.org/10.1080/10656210609484994

Jensen-Campbell, L. A., Graziano, W. G., \& West, S. G. (1995). Dominance, prosocial orientation, and female preferences: Do nice guys really finish last? Journal of Personality and Social Psychology, 68(3), 427-440. https://doi.org/10.1037/0022-3514.68.3.427

John, O. P., \& Srivastava, S. (1999). The Big Five trait taxonomy: History, measurement, and theoretical perspectives. Handbook of Personality: Theory and Research, 2(510), 102-138. https://doi.org/citeulike-article-id:3488537

Judge, T. A., \& Cable, D. M. (1997). Applicant personality, organizational culture, and organization attraction. Personnel Psychology, 50(2), 359-394. https://doi.org/10.1111/j.1744-6570.1997.tb00912.x

Judge, T. A., Erez, A., Bono, J. E., \& Thoresen, C. J. (2002). Are measures of self-esteem, neuroticism, locus of control, and generalized self-efficacy indicators of a common core construct? Journal of Personality and Social Psychology, 83(3), 693-710. https://doi.org/10.1037/0022-3514.83.3.693

Kaare, P. R., Mõttus, R., \& Konstabel, K. (2009). Pathological gambling in Estonia: Relationships with personality, self-Esteem, emotional states and cognitive ability. Journal of Gambling Studies, 25(3), 377-390. https://doi.org/10.1007/s10899-009-9119-y
Keng Cheng, S., \& Law, M. Y. (2015). Mediating Effect of Self-Esteem in the Predictive Relationship of Personality and Academic Self-Handicapping. American Journal of Applied Psychology, 4(3), 51. https:// doi.org/10.11648/j.ajap.s.2015040301.19

Koruklu, N. (2015). Personality and Social Problem-Solving: The Mediating Role of Self-Esteem. Educational Sciences: Theory \& Practice, 15(July 2014), 15-19. https://doi.org/10.12738/estp.2015.2.2601

Lawenius, M., \& Veisson, M. (1996). Personality and Self-Esteem in Parents of Disabled Children: a Comparison Between Estonia and Sweden. Social Behavior and Personality, 24(2), 195-204. https://doi. org/10.2224/sbp.1996.24.2.195

Luan, Z., Poorthuis, A. M. G., Hutteman, R., Asendorpf, J. B., Denissen, J. J. A., \& van Aken, M. A. G. (2018). See me through my eyes: Adolescent - parent agreement in personality predicts later self-esteem development. International Journal of Behavioral Development, 42(1), 17-25. https://doi.org/10.1177/0165025417690263

Luk, C. L., \& Bond, M. H. (1993). Personality variation and values endorsement in Chinese University students. Personality and Individual Differences, 14(3), 429-437. https://doi.org/10.1016/0191-8869(93)90312-Q

Markon, K. E., Krueger, R. F., \& Watson, D. (2005). Delineating the structure of normal and abnormal personality: an integrative hierarchical approach. Journal of Personality and Social Psychology, 88(1), 139-157. https:// doi.org/10.1037/0022-3514.88.1.139

Martínez Arias, M. del R., Hernández, M. V., \& Lloreda, M. J. H. (2006). Psicometría. Madrid: Alianza.

McCrae, R. R., \& Costa, P. T. (2012). Personality in adulthood (2nd ed.). New York: The Guilford Press.

McCrae, R. R., \& Löckenhoff, C. E. (2010). Self Regulation and the Five Factor Model of Personality Traits. In R. Hoyle (Ed.), Handbook of personality and self-regulation (pp. 145-168). West Sussex: Wiley-Blackwell.

McDonald, G., \& Leary, M. R. (2005). Social Exclusion and the Life Course. Psychological Bulletin, 13(2), 231-236.

Michaelides, M. P., Zenger, M., Koutsogiorgi, C., Brähler, E., Stöbel-Richter, Y., \& Berth, H. (2016). Personality correlates and gender invariance of wording effects in the German version of the Rosenberg Self-Esteem Scale. Personality and Individual Differences, 97, 13-18. https://doi. org/10.1016/j.paid.2016.03.011

Mruk, C. J. (2013). Defining Self-Esteem as a Relationship between Competence and Worthiness: How a Two-Factor Approach Integrates the Cognitive and Affective Dimensions of Self-Esteem. Polish Psychological Bulletin, 44(2), 157-164. https://doi.org/10.2478/ppb-2013-0018

Mruk, C. J. (2017). Positive Psychology, Relational Self-Esteem, and Increasing Well-Being. In M. A. Warren \& S. I. Donaldson (Eds.), Toward a Positive Psychology of Relationships: New Directions in Theory and Research (pp. 35-54). Santa Barbara: Praeger.

Neto, F., \& Mullet, E. (2004). Personality, self-esteem, and self-construal as correlates of forgivingness. European Journal of Personality, 18(1), 15-30. https://doi.org/10.1002/per.500 
Paiva, T. T., Pimente, C. E., \& Moura, G. B. de. (2017). Violência conjugal e suas relações com autoestima, personalidade e satisfação com a vida. Gerais: Revista Interinstitucional de Psicologia, 10(2), 215-227.

Piedmont, R. L. (1999). Does Spirituality Represent the Sixth Factor of Personality? Spiritual Transcendence and the Five-Factor Model. Journal of Personality, 67(6), 985-1013. https://doi.org/10.1111/1467-6494.00080

Piedmont, R. L., \& Chae, J.-H. J.-H. (1997). Cross-Cultural Generalizability of the Five-Factor Model of Personality: Development and Validation of the NEO PI-R for Koreans. Journal of Cross-Cultural Psychology, 28(2), 131-155. https://doi.org/10.1177/0022022197282001

Piedmont, R. L., Sherman, M. ., \& Sherman, N. . (2012). Maladaptively high and low openness: the case for experiential permeability. Journal of Personality, 80(6), 1641-1668. https://doi.org/10.1111/ j.14676494.2012.00777.x

Rassart, J., Luyckx, K., Moons, P., \& Weets, I. (2014). Personality and self-esteem in emerging adults with Type 1 diabetes. Journal of Psychosomatic Research, 76(2), 139-145. https://doi.org/10.1016/j. jpsychores.2013.11.015

Rhodewalt, F., \& Tragakis, M. W. (2003). Self-Esteem and Self-Regulation: Toward Optimal Studies of Self-Esteem. Psychological Inquiry, 14(1), 66-70.

Robins, R. W., Tracy, J. L., Trzesniewski, K., Potter, J., \& Gosling, S. D. (2001). Personality correlates of self-esteem. Journal of Research in Personality, 35(4), 463-482. https://doi.org/10.1006/jrpe.2001.2324

Rosenberg, M. (1965). Society and the Adolescent Self-Image. Princeton: Princeton University Press.

Schaffhuser, K., Wagner, J., Lüdtke, O., \& Allemand, M. (2014). Dyadic longitudinal interplay between personality and relationship satisfaction: A focus on neuroticism and self-esteem. Journal of Research in Personality, 53, 124-133. https://doi.org/10.1016/j.jrp.2014.08.007

Schwab, R., \& Petersen, K. U. (1990). Religiousness: Its Relation to Loneliness, Neuroticism and Subjective Well-Being. Journal for the Scientific Study of Religion, 29(3), 335. https://doi.org/10.2307/1386462

Shackelford, T. K., \& Michalski, R. L. (2011). Personality and self-esteem in newlyweds. Personality and Individual Differences, 51(7), 870-872. https://doi.org/10.1016/j.paid.2011.07.018

Shi, M., Liu, L., Yang, Y.-L., \& Wang, L. (2015). The mediating role of self-esteem in the relationship between big five personality traits and depressive symptoms among Chinese undergraduate medical students. Personality and Individual Differences, 83, 55-59. https://doi. org/10.1016/j.paid.2015.03.050

Shikishima, C., Hiraishi, K., Takahashi, Y., Yamagata, S., Yamaguchi, S., \& Ando, J. (2018). Genetic and environmental etiology of stability and changes in self-esteem linked to personality: A Japanese twin study. Personality and Individual Differences, 121, 140-146. https://doi. org/10.1016/j.paid.2017.09.013

Simkin, H. (2013). Personalidad y autoestima en estudiantes universitarios de la Ciudad Autónoma de Buenos Aires. Tesis de Maestría. Universidad Autónoma de Madrid, Madrid.
Simkin, H. (2017). Adaptación y Validación al Español de la Escala de Evaluación de Espiritualidad y Sentimientos Religiosos (ASPIRES): la trascendencia espiritual en el modelo de los cinco factores. Universitas Psychologica, 16(2), 1-12. https://doi.org/10.11144/Javeriana. upsy16-2.aeee

Simkin, H., \& Azzollini, S. (2015). Personalidad, Autoestima y Espiritualidad-Religiosidad desde el Modelo y la Teoría de los Cinco Factores. PSIENCIA: Revista Latinoamericana de Ciencia Psicológica, 7(2), 339-361. https://doi.org/10.5872/psiencia/7.2.22

Şimşek, Ö. F. (2012). Higher-order factors of personality in self-report data: Self-esteem really matters. Personality and Individual Differences, 53(5), 568-573. https://doi.org/10.1016/j.paid.2012.04.023

Swickert, R., Hittner, J. B., Kitos, N., \& Cox-Fuenzalida, L.-E. (2004). Direct or indirect, that is the question: a re-evaluation of extraversion's influence on self-esteem. Personality and Individual Differences, 36(1), 207-217. https://doi.org/10.1016/S0191-8869(03)00080-1

Swickert, R. J., Hittner, J. B., \& Foster, A. (2010). Big Five traits interact to predict perceived social support. Personality and Individual Differences, 48(6), 736-741. https://doi.org/10.1016/j.paid.2010.01.018

Tsigilis, N., \& Srebauite, D. (2015). Dose Self-Esteem Mediates the Association Between Personality Traits and Happiness? A Structural Equation Modeling Approach. Journal of Social Science Studies, 2(2), 102. https://doi.org/10.5296/jsss.v2i2.7370

von Soest, T., Wagner, J., Hansen, T., \& Gerstorf, D. (2017). Self-Esteem Across the Second Half of Life: The Role of Socioeconomic Status, Physical Health, Social Relationships, and Personality Factors. Journal of Personality and Social Psychology, 1(1). https://doi.org/10.1037/ pspp0000123

Wagner, J., \& Gerstorf, D. (2017). Introduction to the special section on self-esteem and personality across the lifespan: Antecedents of development and change. International Journal of Behavioral Development, 42(1), 1-3. https://doi.org/10.1177/0165025416678094

Zeigler-Hill, V., Besser, A., Myers, E. M., Southard, A. C., \& Malkin, M. L. (2013). The Status-Signaling Property of Self-Esteem: The Role of Self-Reported Self-Esteem and Perceived Self-Esteem in Personality Judgments. Journal of Personality, 81(2), 209-220. https://doi. org/10.1111/j.1467-6494.2012.00790.x

Zeigler-Hill, V., Holden, C. J., Enjaian, B., Southard, A. C., Besser, A., Li, H., \& Zhang, Q. (2015). Self-Esteem Instability and Personality: The Connections Between Feelings of Self-Worth and the Big Five Dimensions of Personality. Personality and Social Psychology Bulletin, 41(2), 183-198. https://doi.org/10.1177/0146167214559719

Zitny, P., \& Halama, P. (2011). Self-Esteem, Locus of Control and Personality Traits as Predictors of Sensitivity to Injustice. Studia Psychologica, 1(53), 27-40. 
